\title{
Análise atmosférica em multicamadas em situações de neve no sul do Brasil
}

\author{
Multi-layer atmospheric analysis during snow episodes in southern Brazil
}

\author{
Jéssica Melo Mintegui ${ }^{{ }^{1}}$, Franciano Scremin Puhales ${ }^{2}$, Ernani de Lima Nascimento ${ }^{3}$, \\ Taciana Menezes Weber ${ }^{4}$, Gabriele Golart Silva ${ }^{5}$,Vagner Anabor ${ }^{6}$, Everson Dal Piva ${ }^{7} \mathrm{e}$ \\ Gilsania de Souza Cruz ${ }^{8}$ \\ 1, 2,3,5,6,7 Grupo de Modelagem Atmosférica, Departamento de fisica - Universidade Federal de Santa Maria, Brasil. \\ ${ }^{4}$ Squitter Ambiental, Brasil. \\ ${ }^{8}$ Centro de Informações de Recursos Ambientais e de Hidrometeorologia de Santa Catarina - Empresa de Pesquisa \\ Agropecuária e Extensão Rural de Santa Catarina
}

\begin{abstract}
Resumo
Uma climatologia das variáveis espessura, temperatura, umidades relativa, e conteúdo integrado de vapor d'água é apresentada para diferentes camadas atmosféricas em situações de neve registradas na região sul do Brasil entre 1979 e 2011, totalizando 64 eventos. Na análise empregaram-se dados do "The Climate Forecast System Reanalysis" (CFSR), para as seguintes camadas atmosféricas (em hPa): 850-1000, 700-1000, 500-1000, e 700850. Os episódios de neve foram subdivididos em três grupos de acordo com a altitude da região. A análise das espessuras apresentou valores maiores que os esperados para o Hemisfério Norte. Adicionalmente, a análise do conteúdo integrado de vapor d'água e campos de temperatura e umidade relativa na baixa troposfera, mostrou que a camada atmosférica com condições favoráveis à produção de neve na região sul do Brasil é relativamente rasa, ficando abaixo dos $850 \mathrm{hPa}$. Além disso, os valores limiares de espessura para a ocorrência de neve no sul do Brasil são da ordem de 133 dam para a camada 850-1000 hPa, sendo este valor consideravelmente maior que o documentado para a América do Norte, ressaltando o papel desempenhado pelo efeito orográfico local em criar as condições favoráveis para a ocorrência de neve no Brasil.
\end{abstract}

Palavras-chave: Precipitação de neve. Hemisfério Sul. Sul do Brasil

\begin{abstract}
A climatology for thickness, temperature, absolute and relative humidities, and vertically-integrated water vapor is presented for a number of distinct atmospheric layers during snow episodes reported in Southern Brazil from January 1979 to March 2011, totaling 64 events. Data from "The Climate Forecast System Reanalysis" (CFSR) were used in the analysis of aforementioned variables for the following atmospheric layers: 850-1000 hPa, 700$1000 \mathrm{hPa}, 500-1000 \mathrm{hPa}$, and 700-850 hPa. The snow episodes were subdivided into three sets according to the local surface elevation. The thicknesses of the atmospheric layers found for the snow events in southern Brazil were considerably deeper than the corresponding ones documented in the literature for North America. In addition, analysis of the vertical distribution of water vapor, temperature and relative humidity in the lower troposphere indicated that the atmospheric layer with favorable conditions to snow production is relatively shallow, being below 850hPa. Moreover, the threshold value of thickness for the 850-1000h Palayer during snow occurrence in southern Brazil was around 133 dam, being considerably higher than its counterpart value documented for North America, highlighting the role played by the local topography in setting the conditions that are favorable for snow occurrence in Brazil.
\end{abstract}

Keywords: Snow precipitation. South Hemisphere. Southern of Brazil 


\section{Introdução}

A neve no Brasil é um fenômeno que ocorre princi-palmente nas serras gaúcha e catarinense (Fuen-. tes, 2009). Apesar de ter um impacto econômico positivo para as cidades nas quais ocorre, especialmente pela geração de renda com o turismo e repercussãona mídia, a neve também causa preocupação por seus potenciais prejuízos para a agricultura, transportes e, circulação de veículos, e principalmente pelo fator de risco para as pessoas desprotegidas do frio.

Nos vários casos registrados de neve no sul do Brasil, o acumulado em superfície é baixo. Porém, existem renas cidade de Ijuí-RS, em agosto de 1965 ( Zero (2007)) e superior a $1 \mathrm{~m}$ em São Joaquim-SC em julho de 1957 ( G1 (2014)), por exemplo. Os únicos registros destes casos são fotos e matérias de jornais da época, que não permitem uma avaliação quantitativa dos processos físicos e condições meteorológicas para estes casos.

Diversos estudos já foram realizados para determinar as condições necessárias para a precipitação de neve. Por exemplo Dewey (1977); Heppner (1992); Johnston (1995), contudo, a maioria deles concentra-se especificamente para o hemisfério norte e, a simples transposição desses modelos pode não ser eficiente para a previsão de neve no nosso hemisfério. Neste ponto se faz uma ressalva ao importante trabalho de Fuentes (2009), que investigou a dinâmica e os padrões de circulação atmosférica associadas a eventos de precipitação de neve no sul do Brasil.

O presente estudo tem como principal objetivo desenvolver uma análise da espessura e água precipitável de determinadas camadas atmosféricas. Tal análise visa aprimorar a previsão de precipitação de neve na região Sul do Brasil. Além disso, foi realizada uma avaliação da umidade relativa e temperatura média nas camas de 850 $\mathrm{hPa}$ e $700 \mathrm{hPa}$. Estas duas grandezas são importantes pois evidenciam os dois fatores preponderantes para a formação de neve: baixas temperaturas e disponibilidade de umidade. Os dados empregados foram aqueles gerados a partir da The Climate Forecast System Reanalysis, com uma resolução temporal de seis horas e espacial de meio grau (Saha et al., 2010).

\section{Materiais e Métodos}

A ideia base deste trabalho é a análise de espessura e da água precipitável em distintas camadas da atmosfera. As camadas utilizadas foram as mesmas propostas por latos de acumulo de neve maior que dois metros, como

(Weber, 2011). Além disso, também se considera a temperatura e umidade relativa nos níveis de $850 \mathrm{hPa}$ e 700 $\mathrm{hPa}$.

Os dados utilizados para o estudo são os da CFSR (The Climate Forecast System Reanalysis) (Saha et al., 2010). De forma geral, os dados de reanálise são formados a partir de assimilação de dados observados com dados de um modelo de previsão, que são disponibilizados em uma grade regular no espaço e em intervalos de tempo constantes. Como as observações não são realizadas em uma grade regular e nem sempre são obtidas nos mesmos instantes de tempo, o sistema de assimilação de dados combina as informações observacionais com os dados de modelo, gerando campos meteorológicos em uma grade espacial e intervalos de tempo regulares (Quadro, 2012). Ainda, Silva et al. (2011 apud Quadro, 2012) cita as principais diferenças entre a CFSR e as demais reanálises, como por exemplo a NCEP-DOE-R2:

(i) alta resolução vertical e horizontal;

(ii) o modelo utilizado inclui o acoplamento entre continentes, atmosfera, oceanos e superfícies cobertas por gelo;

(iii) a assimilação de grandezas radiativas médias, obtidas a partir de dados de satélite.

Os dados de da CFSR (Saha et al., 2010) possuem uma resolução espacial horizontal $0,5^{\circ} \times 0,5^{\circ}$, divididos em 37 níveis verticais isobáricos ${ }^{1}$ e resolução temporal de seis horas. Nesta grade são disponibilizadas mais de 200 variáveis meteorológicas ${ }^{2}$, tais como: altura geopotencial, temperatura, umidade específica, etc., incluindo variáveis de superfície como pressão reduzida ao nível do mar, temperatura e umidade relativa a $2 \mathrm{~m}$. Para todo o globo, o arquivo possui 720 pontos em longitude e 181 pontos de latitude.

Devido a melhor resolução espacial dos dados de CFSR, em relação aos de $\mathrm{R} 2^{3}$, há vantagem de se utilizar tais dados para uma melhor discretização das variáveis. Além do mais, este conjunto de dados conta com uma assimilação observacional mais eficiente, uma vez que conta com dados de acoplamento entre atmosfera e oceano. Como desvantagem, a CFSR apresenta um período limitado de dados: de janeiro de 1979 até março de 2011. Por sua vez, os dados de $2,5^{\circ}$ são disponibilizados de forma operacional, com período de janeiro de 1979 até o mês atual.

A espessura de cada camada é definida como:

$$
\delta Z=Z_{p-\delta p}-Z_{p}
$$

${ }^{1}$ Os níveis isobáricos são (em hPa): 1000, 975, 950, 925, 900, 875, 850, 825, 800, 775, 750, 700, 650, 600, 550, 500, 450, 400, 350, 300, 250, 225, 200, 175, 150, 125, 100, 70, 50, 30, 20, 10, 7, 5, 3, 2 e 1 .

${ }^{2} \mathrm{~A}$ lista completa das variáveis pode ser obtida em http://rda.ucar.edu/datasets/dsC93.C/\#description

${ }^{3} \mathrm{O}$ conjunto de dados da NCEP-DOE-R2 apresenta resolução horizontal de 2,50 em 28 níveis isobáricos entre $1000 \mathrm{hPa}$ e $10 \mathrm{hPa}$ 
onde $Z_{p}$ é a altura geopotencial em uma superfície isobárica $p$ e $\delta p>0$ é a diferença entre duas superfícies isobáricas. A partir dos dados de espessura foi possível estimar a temperatura virtual média da camada. Assumindo-se a aproximação hidrostática em cada uma das camadas apresentadas na tabela 1 e a equação dos gases ideais, pode-se escrever:

Tabela 1: Variáveis analisadas no estudo.

\begin{tabular}{llr}
\hline Nível & Símbolo & Unidade \\
\hline $500-1000 \mathrm{hPa}$ & $\delta Z$ e $W_{p}$ & dam e $k g / m^{2}$ \\
$700-1000 \mathrm{hPa}$ & $\delta Z$ e $W_{p}$ & $d a m$ e $k g / m^{2}$ \\
$850-1000 \mathrm{hPa}$ & $\delta Z$ e $W_{p}$ & $d a m$ e $k g / m^{2}$ \\
$700-850 \mathrm{hPa}$ & $\delta Z$ e $W_{p}$ & dam e $k g / m^{2}$ \\
$850 \mathrm{hPa}$ & $T_{850}$ e $U r_{850}$ & $K$ e $\%$ \\
$700 \mathrm{hPa}$ & $T_{700}$ e $U r_{700}$ & $K$ e $\%$ \\
\hline
\end{tabular}

$$
\frac{d p}{p}=-\frac{1}{R_{d} T_{v}} d z
$$

onde $\rho$ é a densidade do ar, $R_{d}=287 \mathrm{~J} \mathrm{~kg}^{-1} \mathrm{~K}^{-1}, T_{v}$ é a temperatura virtual, $g=9,81 \mathrm{~m} \mathrm{~s}^{-2}$ é a aceleração da gravidade e $z$ é a coordenada vertical orientada para cima. Assumindo que a temperatura na equação 2 é a temperatura média da camada, $T_{v} \equiv\left\langle T_{v}\right\rangle$ é uma constante, onde o operador $\langle\cdots\rangle$ representa uma média ao longo do perfil vertical da camada analisada. Desta forma, da equação 2 resulta:

$$
\begin{aligned}
\int_{p}^{p-\delta p} \frac{d p}{p} & =-\frac{1}{R_{d}\left\langle T_{v}\right\rangle} \int_{Z_{p}}^{Z_{p-\delta p}} d z \\
\left\langle T_{v}\right\rangle & =-\frac{\delta Z}{R_{d}}\left[\ln \left(\frac{p-\delta p}{p}\right)\right]^{-1}
\end{aligned}
$$

A água precipitável $W_{p}$ em cada camada é definida por:

$$
W_{p}=\frac{1}{g} \int_{p}^{p_{0}} q d p
$$

onde $q$ é a umidade específica, e $p_{0}$ e $p$ delimitam a camada atmosférica analisada.

Os eventos de precipitação de neve (data e local) foram obtidos a partir do trabalho de (Carvalho Júnior, 2004), o qual faz uma analise histórica dos eventos de neve principalmente para a região sul do Brasil. Tal estudo compreende o período de 1923 até 2000. Devido a limitação dos dados foram analisadas as datas a partir de 1979. A lista foi complementada com o registro histórico fornecido pelo Epagri/Ciram (CIRAM, 2014; Cruz,
2014). Esta complementação continha dados referentes ao estado de Santa Catarina, no período de 1999 a 2014. Novamente, foram utilizados os casos até março de 2011. Com base nestes históricos foram separados 64 eventos de neve, em diferentes pontos da região sul do Brasil.

Os casos foram agrupados em cidades representativas de uma região. Além do mais, as cidades representativas foram separadas em três grupos, utilizando como critério de divisão a altitude em que cada cidade está situada no dado de topografia do CFSR. A tabela 2 apresenta as cidades representativas em cada grupo do estudo, enquanto a figura 1 mostra a posição geográfica das mesmas. Cada um dos casos contribuiu, em geral, com mais de uma amostra devido a ocorrência de neve em regiões distintas no mesmo período. O grupo 1 (G1) foram contabilizados 14 amostras, enquanto nos grupos 2 (G2) e 3 (G3) 35 e 65 respectivamente.

O domínio utilizado é dado pelas seguintes coordenadas geográficas: $15^{\circ} \mathrm{S}$ à $55^{\circ} \mathrm{S}$ de latitude e $75^{\circ} \mathrm{W}$ à $5^{\circ} \mathrm{W}$ de longitude. Esta área serviu para a avaliação dos sistemas sinóticos que atuavam sobre os estados ou que influenciavam os mesmos de alguma forma. Uma região menor foi escolhida, com latitude de $20^{\circ} \mathrm{S}$ à $40^{\circ} \mathrm{S}$ e longitude de $75^{\circ} \mathrm{W}$ à $45^{\circ} \mathrm{W}$, para análise da umidade, somente sobre a região de estudo.

Com isso, foi realizado um estudo estatístico em termos das amostras. Cada amostra foi computada dentro de seu grupo nos horários UTC de 00Z, 06Z, 12Z e 18Z. A fim de obter parâmetros que representem estatisticamente os casos foram construídos diagramas de caixa para os grupos de amostras. Os diagramas de caixa dão uma ideia geral da distribuição estatística dos dados (McGill et al., 1978; Wilks, 2006).

\section{Resultados e Discussões}

Para cada um dos 64 casos selecionados foram analisados os campos de espessura e água precipitável em diferentes camadas da atmosfera, além dos campos de temperatura e umidade relativa nos níveis de 850 e $700 \mathrm{hPa}$.

A figura 2 apresenta o diagrama de caixas para a espessura das diferentes camadas, horários e grupos. É possível observar que não há grande diferença entre os valores médios das medianas entre os grupos. Além do mais, a distribuição das amostras em termos dos quantis é bastante semelhante entre os diferentes horários do mesmo grupo. Adicionalmente, tal semelhança se mantém entre G2 e G3. O G1, para todas as camadas, é o que apresenta maior variabilidade. Contudo, tal grupo, é o que possui a menor amostra de casos. Além disso, na figura 2 é possível notar que existem valores atípicos nos valores de espessura. Porém, para este trabalho, os valores atípicos mínimos de espessura, além de serem raros, não influenciam esta análise, pois o que se espera é en- 
contrar um valor máximo de espessura que seja propício à precipitação de neve. Os valores atípicos superiores, mesmo que existentes, apresentam uma pequena ocorrência, e a pequena diferença entre os extremos confere a amostra um pequeno espalhamento estatístico. Além do mais, os valores mínimos e máximos da amostra são em geral menores que os limites estatísticos típicos.

Tabela 2: Grupos de cidades

\begin{tabular}{clccc}
\hline Grupo & Cidades & Latitude & Longitude & Altitude \\
\hline \multirow{2}{*}{1} & Santa Maria & -29.7 & 307. & \\
& Porto Alegre & -30 & 308. & $h<300 m$ \\
& Bagé & -31.3 & 305.9 & \\
& Quaraí & -30.3 & 303.5 & \\
\hline \multirow{2}{*}{2} & Ijuí & -28.4 & 306.1 & \\
& Caxias do Sul & -29.1 & 308.9 & \\
& São Miguel do Oeste & -26.7 & 306.1 & \multirow{2}{*}{$300 \leq h<800 m$} \\
& Curitiba & -25.4 & 310.7 & \\
& Pato Branco & -26.2 & 307.3 & \\
& Cascavel & -24.9 & 306.5 & \\
\hline \multirow{2}{*}{3} & São José dos Ausentes & -28.7 & 306.1 & \\
& São Joaquim & -28.3 & 310 & \\
& Urupema & -27.9 & 310.2 & \\
& Palmas & -26.5 & 308 & \\
\hline
\end{tabular}

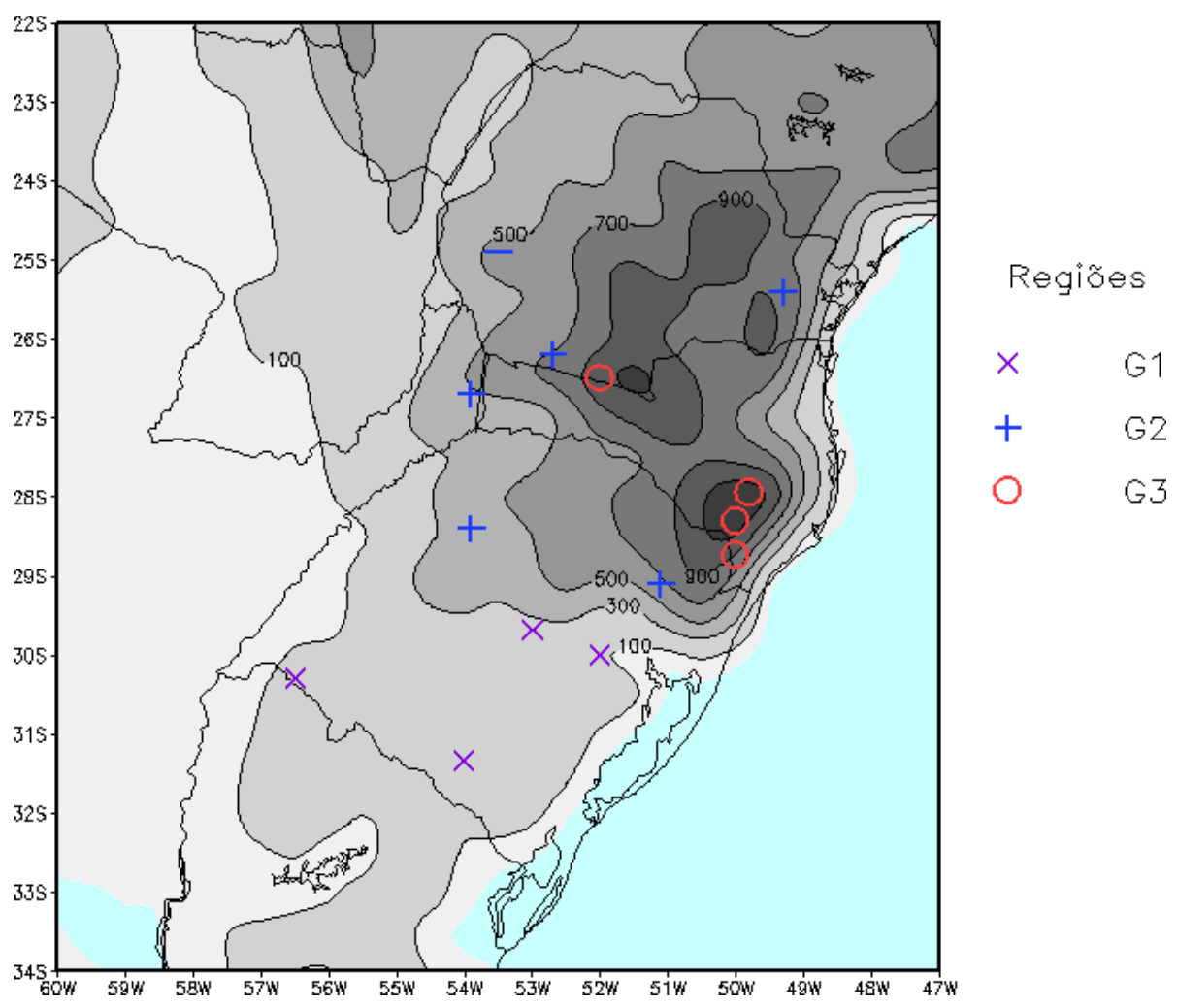

Figura 1: Mapa com os três grupos e cidades correspondentes, a topografia em metros é representada pelas isolinhas. 

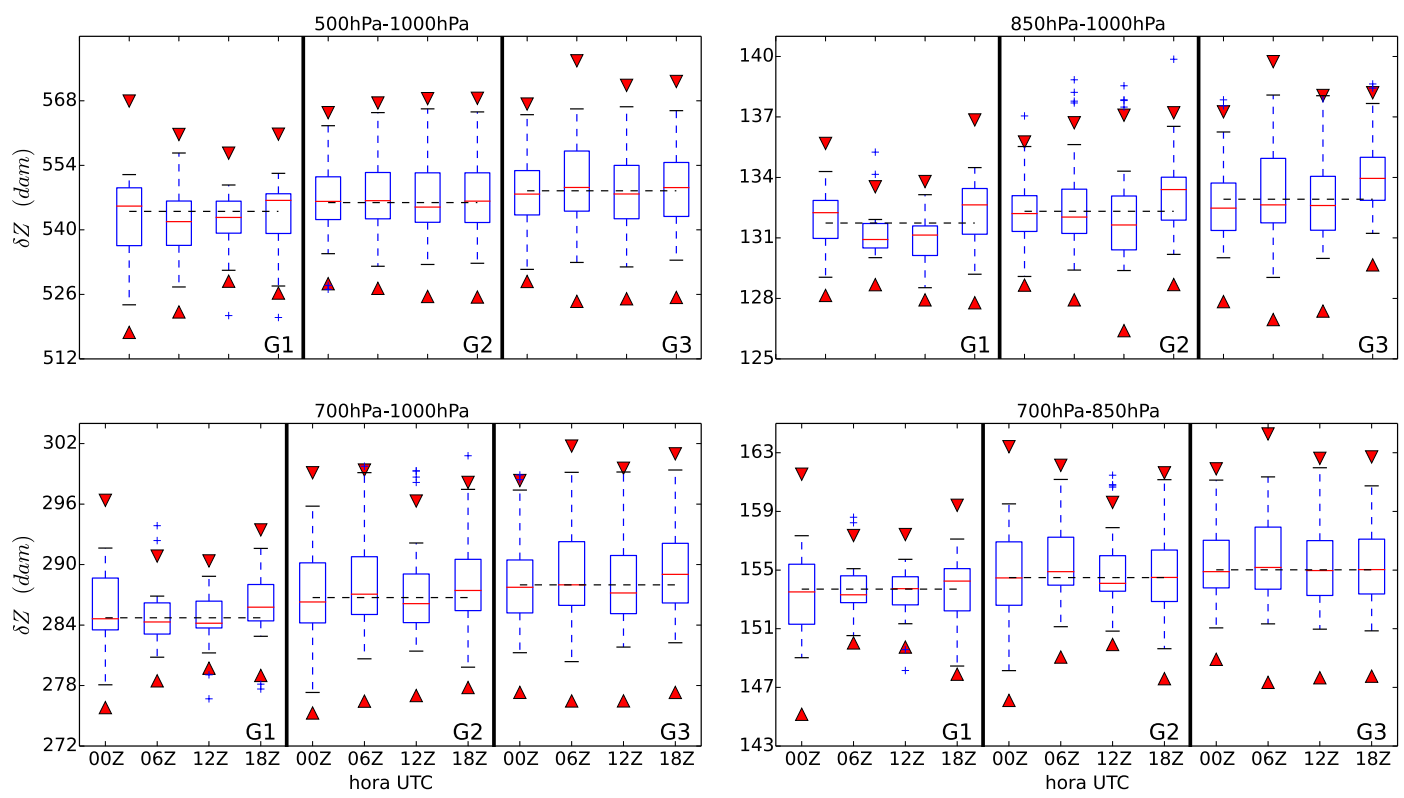

Figura 2: Diagrama de caixa da espessura média da camada para cada grupo. As linhas que definem as bordas inferior e superior da caixa representam, respectivamente, o primeiro e o terceiro quantil da amostra e a linha interna o segundo quantil (mediana). As linhas tracejadas verticais indicam os valores compreendidos no intervalo de confiança da amostra, sendo os seus valores máximo e mínimo representados pelo traço horizontal. O intervalo de confiança é identificado pelos triângulos e os pontos em forma de cruz apresentam valores fora do intervalo de confiança (outliers).

Tabela 4: Valores médios de espessura e temperatura virtual da camada por grupo para a camada entre $500 \mathrm{hPa}$ e $1000 \mathrm{hPa}$. O símbolo X é a variável estatística da grandeza em questão enquanto o operador $\widetilde{(. \cdot)}$ representa a mediana e $\overline{(\cdot \cdot)}$ a média dos quatro horários para cada grupo. Os subíndices $s, i, 25$ e 75 , denotam respectivamente o limite de confiança superior, o limite de confiança inferior, o primeiro quantil e o terceiro quantil da amostra. Os valores classificados como "Geral"são a média dos três grupos.

\begin{tabular}{cccc}
\hline Grupo & Variável estatística & Espessura $($ dam $)$ & Temperatura virtual $\left({ }^{\circ} \mathrm{C}\right)$ \\
\hline \multirow{4}{*}{ Grupo 1 } & $\overline{\widetilde{X}}$ & 544 & $-4,9$ \\
& $\overline{X_{s}}$ & 562 & 3,8 \\
& $\overline{X_{i}}$ & 523 & $-14,9$ \\
& $\overline{X_{25}}$ & 538 & $-7,9$ \\
\hline \multirow{4}{*}{ Grupo 2 } & $\overline{X_{75}}$ & 547 & $-3,3$ \\
& $\overline{\widetilde{X}}$ & 546 & $-3,9$ \\
& $\overline{X_{s}}$ & 567 & 6,7 \\
& $\overline{X_{i}}$ & 527 & $-13,4$ \\
& $\overline{X_{25}}$ & 542 & $-5,9$ \\
Grupo 3 & $\bar{X}_{75}$ & 552 & $-0,8$ \\
& $\overline{\widetilde{X}}$ & 548 & $-2,7$ \\
& $\overline{X_{s}}$ & 571 & 8,9 \\
& $\overline{X_{i}}$ & 526 & $-13,8$ \\
& $\overline{X_{25}}$ & 543 & $-5,3$ \\
Geral & $X_{75}$ & 555 & 0,4 \\
\hline \multirow{3}{*}{} & $\overline{\widetilde{X}}$ & 546 & $-3,8$ \\
& $\overline{X_{s}}$ & 567 & 6,4 \\
& $\overline{X_{i}}$ & 525 & $-14,0$ \\
& $\overline{X_{25}}$ & 541 & $-6,3$ \\
$X_{75}$ & 551 & $-1,2$ \\
\hline
\end{tabular}


Tabela 5: O mesmo que a tabela 4 para a camada entre $850 \mathrm{hPa}$ e $1000 \mathrm{hPa}$.

\begin{tabular}{cccc}
\hline Grupo & Variável estatística & Espessura (dam) & Temperatura virtual $\left({ }^{\circ} \mathrm{C}\right)$ \\
\hline \multirow{3}{*}{ Grupo 1 } & $\overline{\widetilde{X}}$ & 132 & 3,9 \\
& $\overline{X_{s}}$ & 135 & 10,7 \\
& $\overline{X_{i}}$ & 128 & $-3,6$ \\
& $\overline{X_{25}}$ & 130 & 1,73 \\
Grupo 2 & $\overline{X_{75}}$ & 132 & 5,3 \\
\hline \multirow{3}{*}{ Grupo 3 } & $\overline{\widetilde{X}}$ & 132 & 5,1 \\
& $\overline{X_{s}}$ & 137 & 14,3 \\
& $\overline{X_{i}}$ & 128 & $-4,1$ \\
& $\overline{X_{25}}$ & 131 & 2,8 \\
& $\overline{X_{75}}$ & 133 & 7,4 \\
\hline \multirow{3}{*}{ Geral } & $\overline{\widetilde{X}}$ & 132 & 6,4 \\
& $\overline{X_{s}}$ & 138 & 17,7 \\
& $\overline{X_{25}}$ & 128 & $-4,0$ \\
& $\overline{X_{75}}$ & 132 & 4,1 \\
& $\overline{\widetilde{X}}$ & 134 & 9,5 \\
\hline$X_{s}$ & 132 & 5,2 \\
& $\overline{X_{i}}$ & 137 & 14,3 \\
& $\overline{X_{25}}$ & 128 & $-3,8$ \\
$X_{75}$ & 131 & 2,9 \\
\hline
\end{tabular}

Tabela 6: O mesmo que a tabela 4 para a camada entre $700 \mathrm{hPa}$ e $1000 \mathrm{hPa}$.

\begin{tabular}{|c|c|c|c|}
\hline Grupo & Variável estatística & Espessura (dam) & Temperatura virtual $\left({ }^{\circ} \mathrm{C}\right)$ \\
\hline \multirow{5}{*}{ Grupo 1} & $\overline{\widetilde{X}}$ & 285 & $-0,3$ \\
\hline & $\overline{X_{S}}$ & 293 & 7,4 \\
\hline & $\overline{X_{i}}$ & 278 & $-6,5$ \\
\hline & $\overline{X_{25}}$ & 284 & $-1,28$ \\
\hline & $\overline{X_{75}}$ & 287 & 2,19 \\
\hline \multirow{5}{*}{ Grupo 2} & $\widetilde{\widetilde{X}}$ & 287 & 1,6 \\
\hline & $\overline{X_{s}}$ & 298 & 12,6 \\
\hline & $\overline{X_{i}}$ & 277 & $-8,0$ \\
\hline & $\overline{X_{25}}$ & 285 & $-0,3$ \\
\hline & $\overline{X_{75}}$ & 290 & 4,9 \\
\hline \multirow{5}{*}{ Grupo 3} & $\underline{\widetilde{X}}$ & 288 & 2,8 \\
\hline & $\overline{X_{s}}$ & 300 & 14,5 \\
\hline & $\overline{X_{i}}$ & 277 & $-7,8$ \\
\hline & $\overline{X_{25}}$ & 285 & 0,6 \\
\hline & $\overline{X_{75}}$ & 291 & 6,1 \\
\hline \multirow{5}{*}{ Geral } & $\overline{\widetilde{X}}$ & 286 & 1,4 \\
\hline & $\overline{X_{s}}$ & 297 & 11,5 \\
\hline & $\overline{X_{i}}$ & 277 & $-7,4$ \\
\hline & $\overline{X_{25}}$ & 285 & $-0,3$ \\
\hline & $\overline{X_{75}}$ & 290 & 4,4 \\
\hline
\end{tabular}


Tabela 7: O mesmo que a tabela 4 para a camada entre $700 \mathrm{hPa}$ e $850 \mathrm{hPa}$.

\begin{tabular}{cccc}
\hline Grupo & Variável estatística & Espessura (dam) & Temperatura virtual $\left({ }^{\circ} \mathrm{C}\right)$ \\
\hline \multirow{4}{*}{ Grupo 1 } & $\overline{\widetilde{X}}$ & 154 & $-2,5$ \\
& $\overline{X_{s}}$ & 159 & 6,8 \\
& $\overline{X_{i}}$ & 148 & $-12,2$ \\
& $\overline{X_{25}}$ & 152 & $-5,1$ \\
Grupo 2 & $\overline{X_{75}}$ & 155 & $-0,4$ \\
& $\overline{\widetilde{X}}$ & 154 & $-1,2$ \\
& $\overline{X_{s}}$ & 162 & 11,5 \\
& $\overline{X_{i}}$ & 148 & $-12,3$ \\
& $\overline{X_{25}}$ & 153 & $-3,3$ \\
Grupo 3 & $\overline{X_{75}}$ & 157 & 2,6 \\
& $\overline{\widetilde{X}}$ & 155 & $-0,2$ \\
& $\overline{X_{s}}$ & 163 & 13,5 \\
& $\overline{X_{i}}$ & 148 & $-12,7$ \\
& $\overline{X_{25}}$ & 153 & $-2,9$ \\
\hline \multirow{3}{*}{ Geral } & $\overline{X_{75}}$ & 157 & 1,9 \\
& $\overline{\widetilde{X}}$ & 154 & $-1,3$ \\
& $\overline{X_{s}}$ & 161 & 10,5 \\
& $\overline{X_{i}}$ & 148 & $-12,4$ \\
& $\overline{X_{25}}$ & 153 & $-3,8$ \\
\hline & $X_{75}$ & 156 & 2,0 \\
\hline
\end{tabular}

As tabelas 4, 5, 6 e 7, sumarizam os resultados do diagrama de caixa para as diferentes espessuras. De forma complementar, utilizando-se a equação 3 foi calculado um valor médio para a temperatura de cada camada, também apresentado nas tabelas.

Os resultados obtidos para os diagramas de caixa da água precipitável são mostrados na figura 3. De forma geral essa variável apresenta um maior espalhamento estatístico e valores além daquelas considerados típicos, quando comparados a aqueles obtidos para a espessura das diferentes camadas. Além disso, há uma maior variação entre os grupos e horários, sendo que esta variação é mais fortemente percebida no G1. Nesta análise, um objetivo importante é reconhecer um valor típico mínimo de água precipitável em cada camada para a ocorrência de neve. Tal limite não é tão confiável, do ponto de vista estatístico, como o encontrado para a espessura, porém pode dar um indício inicial para uma previsão objetiva do fenômeno. Além do mais os dados de umidade assimilados pela reanálise não são tão confiáveis quanto os de altura geopotencial, devido a forte influência local (em uma escala menor que a resolução da grade) neste variável. As tabelas 8, 9, 10 e 11, sumarizam os resultados dos diagramas de caixa para a água precipitável.

A figura 4 mostra o diagrama de caixas para a temperatura e umidade relativa nos níveis de $850 \mathrm{hPa}$ e 700
hPa. A partir desta pode-se observar que no nível de 850 $\mathrm{hPa}$, os valores de temperatura típicos, assim como as médias das medianas possuem valores menores para o G1, região mais baixa, e os valores para os demais grupos ficaram muito próximos, o mesmo se vê em $700 \mathrm{hPa}$, onde os valores de G2 e G3 se assemelham muito. A análise da umidade relativa em $850 \mathrm{hPa}$ e $700 \mathrm{hPa}$, não se mostra conclusiva, pois não existem valores máximos ou mínimos em comum, exibindo um grande espalhamento estatístico da amostra. Como umidade relativa alta e baixa temperatura são parâmetros necessários para a ocorrência de neve, pode-se inferir que os fenômenos de ocorrência de neve no sul do Brasil ocorrem em camadas rasas, e por vezes mais baixas que $850 \mathrm{hPa}$. Assim, para alguns casos analisados a neve pode ter se formado abaixo dos $700 \mathrm{hPa}$ e até dos $850 \mathrm{hPa}$, gerando o espalhamento estatístico observado. Um outro fator a ser considerado é a qualidade da representação dos dados de umidade no conjunto de dados. Desta forma, a análise de espessura e água precipitável se mostra mais precisa para uma previsão objetiva da ocorrência de neve. As tabelas 12, 13, sumarizam os resultados do diagrama de caixa para temperatura e umidade relativa nos níveis de $850 \mathrm{hPa}$ e $700 \mathrm{hPa}$. 

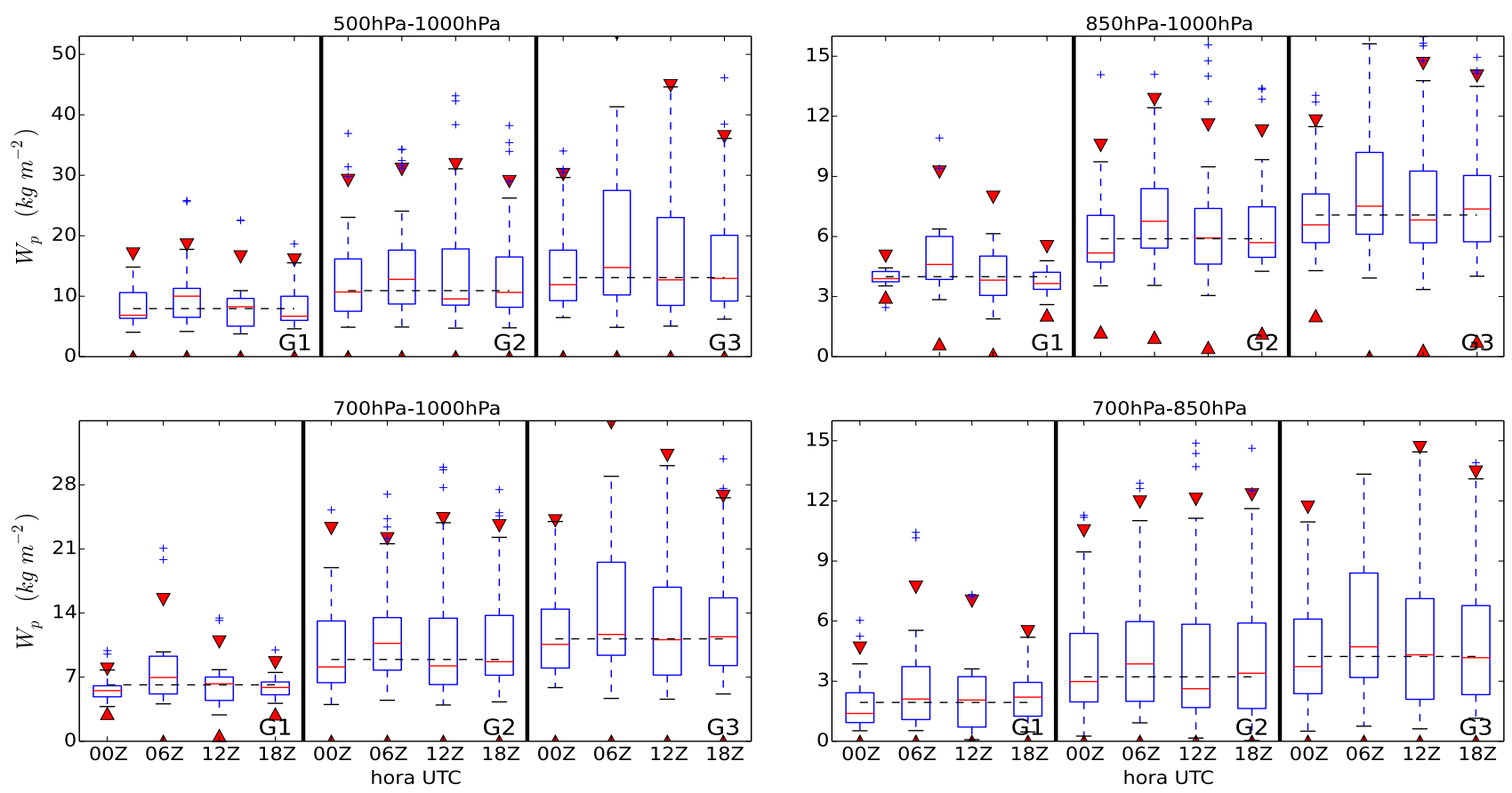

Figura 3: A mesma representação da figura 2, porém para água precipitável.
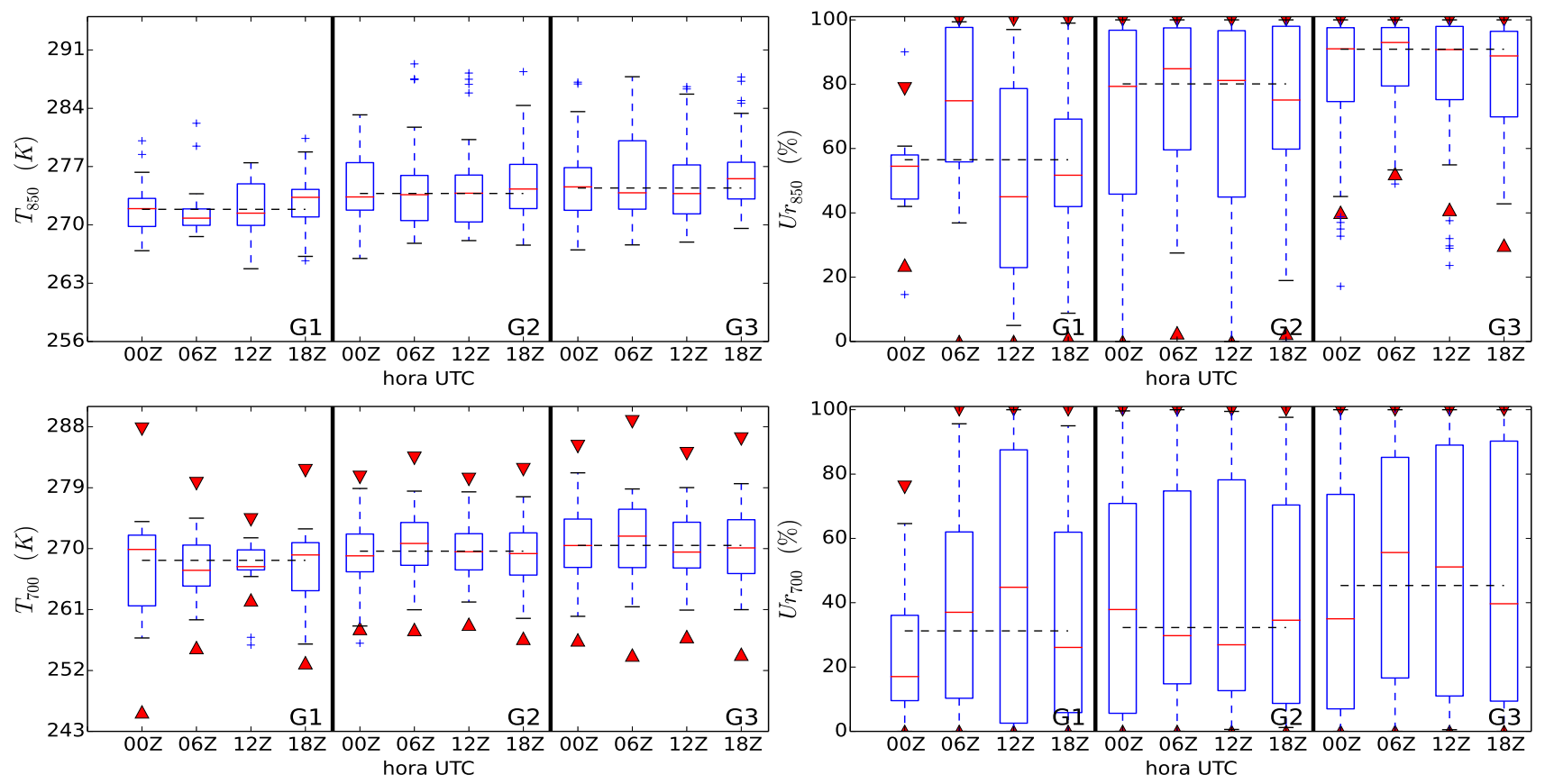

Figura 4: Diagrama de caixa da temperatura e umidade relativa em $850 \mathrm{hPa}$ e $700 \mathrm{hPa}$. 
Tabela 8: Valores médios de água precipitável por grupo para a camada entre $500 \mathrm{hPa}$ e $1000 \mathrm{hPa}$. O operador $\overline{(\cdots)}$ representa a média dos quatro horários para cada grupo. Os valores classificados como "Geral" são a média dos três grupos.

\begin{tabular}{ccc}
\hline Grupo & Variável estatística & Água Precipitável $\left(\mathrm{kgm}^{-2}\right)$ \\
\hline \multirow{3}{*}{ Grupo 1 } & $\overline{\widetilde{X}}$ & 7,9 \\
& $\overline{X_{s}}$ & 17,0 \\
& $\overline{X_{i}}$ & 0,0 \\
& $\overline{X_{25}}$ & 5,9 \\
$\bar{X}_{75}$ & 10,4 \\
\hline \multirow{3}{*}{ Grupo 2 } & $\overline{\widetilde{X}}$ & 10,9 \\
& $\overline{X_{s}}$ & 30,2 \\
& $\overline{X_{i}}$ & 0,0 \\
Grupo 3 & $\overline{X_{25}}$ & 8,2 \\
& $\overline{X_{75}}$ & 17,0 \\
\hline \multirow{3}{*}{ Geral } & $\overline{\widetilde{X}}$ & 13,0 \\
& $\overline{X_{s}}$ & 41,2 \\
& $\overline{X_{i}}$ & 0,0 \\
& $\overline{X_{25}}$ & 9,3 \\
& $\bar{X}_{75}$ & 22,0 \\
\hline & $\overline{\widetilde{X}}$ & 10,6 \\
& $\overline{X_{s}}$ & 29,5 \\
& $\overline{X_{i}}$ & 0,0 \\
\hline & $\overline{X_{75}}$ & 7,8 \\
\hline
\end{tabular}

Tabela 9: O mesmo que a tabela 8 para a camada entre $850 \mathrm{hPa}$ e $1000 \mathrm{hPa}$.

\begin{tabular}{ccc}
\hline Grupo & Variável estatística & Água Precipitável $\left(\mathrm{kgm}^{-2}\right)$ \\
\hline \multirow{3}{*}{ Grupo 1 } & $\overline{\widetilde{X}}$ & 4,0 \\
& $\overline{X_{s}}$ & 6,9 \\
& $\overline{X_{i}}$ & 1,5 \\
& $\overline{X_{25}}$ & 3,5 \\
Grupo 2 & $\overline{X_{75}}$ & 4,9 \\
\hline \multirow{3}{*}{ Grupo 3 } & $\overline{\widetilde{X}}$ & 5,9 \\
& $\overline{X_{s}}$ & 11,6 \\
& $\overline{X_{i}}$ & 1,0 \\
& $\overline{X_{25}}$ & 4,9 \\
& $\overline{X_{75}}$ & 7,6 \\
\hline \multirow{3}{*}{ Geral } & $\overline{X_{s}}$ & 7,1 \\
& $\overline{X_{i}}$ & 14,2 \\
& $\overline{X_{25}}$ & 0,8 \\
& $\bar{X}_{75}$ & 5,8 \\
& $\overline{\widetilde{X}}$ & 9,2 \\
\hline$X_{s}$ & 5,7 \\
& $\overline{X_{i}}$ & 10,9 \\
\hline
\end{tabular}


Tabela 10: O mesmo que a tabela 8 para a camada entre $700 \mathrm{hPa}$ e $1000 \mathrm{hPa}$.

\begin{tabular}{ccc}
\hline Grupo & Variável estatística & Água Precipitável $\left(\mathrm{kgm}^{-2}\right)$ \\
\hline \multirow{3}{*}{ Grupo 1 } & $\overline{\widetilde{X}}$ & 6,2 \\
& $\overline{X_{s}}$ & 10,7 \\
& $\overline{X_{i}}$ & 1,7 \\
& $\overline{X_{25}}$ & 4,9 \\
$\overline{X_{75}}$ & 7,2 \\
\hline \multirow{3}{*}{ Grupo 2 } & $\overline{\widetilde{X}}$ & 8,9 \\
& $\overline{X_{s}}$ & 23,3 \\
& $\overline{X_{i}}$ & 0,0 \\
& $\overline{X_{25}}$ & 6,9 \\
Grupo 3 & $\bar{X}_{75}$ & 13,5 \\
& $\overline{\widetilde{X}}$ & 11,2 \\
& $\overline{X_{s}}$ & 29,2 \\
& $\overline{X_{i}}$ & 0,0 \\
& $\overline{X_{25}}$ & 8,2 \\
Geral & $X_{75}$ & 16,6 \\
& $\overline{\widetilde{X}}$ & 8,8 \\
& $\overline{X_{s}}$ & 21,1 \\
& $\overline{X_{i}}$ & 0,6 \\
& $\overline{X_{25}}$ & 6,7 \\
\hline
\end{tabular}

Tabela 11: O mesmo que a tabela 8 para a camada entre $700 \mathrm{hPa}$ e $1000 \mathrm{hPa}$.

\begin{tabular}{ccc}
\hline Grupo & Variável estatística & Água Precipitável $\left(\mathrm{kgm}^{-2}\right)$ \\
\hline \multirow{3}{*}{ Grupo 1 } & $\overline{\widetilde{X}}$ & 1,9 \\
& $\overline{X_{s}}$ & 6,2 \\
& $\overline{X_{i}}$ & 0,0 \\
& $\overline{X_{25}}$ & 1,0 \\
$\bar{X}_{75}$ & 3,1 \\
\hline \multirow{3}{*}{ Grupo 2 } & $\overline{\widetilde{X}}$ & 3,2 \\
& $\overline{X_{s}}$ & 11,7 \\
& $\overline{X_{i}}$ & 0,0 \\
& $\overline{X_{25}}$ & 1,8 \\
Grupo 3 & $\overline{X_{75}}$ & 5,8 \\
& $\overline{\widetilde{X}}$ & 4,2 \\
& $\overline{X_{s}}$ & 14,0 \\
& $\overline{X_{i}}$ & 0,0 \\
& $\overline{X_{25}}$ & 2,5 \\
Geral & $\bar{X}_{75}$ & 7,1 \\
& $\overline{\widetilde{X}}$ & 3,1 \\
& $\overline{X_{s}}$ & 10,6 \\
& $\overline{X_{i}}$ & 0,0 \\
& $\overline{X_{25}}$ & 1,7 \\
\hline
\end{tabular}


Tabela 12: Valores médios de temperatura e umidade renlativa no nível de $850 \mathrm{hPa}$. O operador $\overline{(\cdots)}$ representa a média dos quatro horários para cada grupo. Os valores classificados como "Geral"são a média dos três grupos.

\begin{tabular}{cccc}
\hline Grupo & Variável estatística & Temperatura $\left({ }^{\circ} \mathrm{C}\right)$ & Umidade relativa (\%) \\
\hline \multirow{3}{*}{ Grupo 1 } & $\overline{\widetilde{X}}$ & $-1,3$ & 56,5 \\
& $\overline{X_{s}}$ & 5,6 & 94,6 \\
& $\overline{X_{i}}$ & $-8,1$ & 6,2 \\
& $\overline{X_{25}}$ & 2,9 & 41,3 \\
Grupo 2 & $\overline{X_{75}}$ & $-0,5$ & 76,1 \\
\hline \multirow{6}{*}{ Grupo 3 } & $\overline{\widetilde{X}}$ & 0,8 & 80,1 \\
& $\overline{X_{s}}$ & 11,8 & 100 \\
& $\overline{X_{i}}$ & $-10,3$ & 1,3 \\
& $\overline{X_{25}}$ & 1,8 & 52,5 \\
& $\overline{X_{75}}$ & $-3,6$ & 97,2 \\
\hline \multirow{3}{*}{ Geral } & $\bar{X}$ & 0,6 & 90,8 \\
& $\overline{X_{s}}$ & 11,8 & 100 \\
& $\overline{X_{i}}$ & $-10,3$ & 40,8 \\
& $\overline{X_{25}}$ & 1,0 & 75,1 \\
& $\overline{X_{75}}$ & $-4,9$ & 97,4 \\
\hline & $\bar{X}$ & 0,2 & 75,8 \\
& $\overline{X_{s}}$ & 10,3 & 98,2 \\
& $\overline{X_{i}}$ & $-9,4$ & 16,1 \\
& $\overline{X_{25}}$ & 1,9 & 56,2 \\
\hline
\end{tabular}

\section{Conclusões}

O presente trabalho se propunha a encontrar valores característicos para espessura e água precipitável em diferentes camadas da atmosfera, quando há ocorrência de neve. Além disso, valores típicos de temperatura e umidade relativa nos níveis verticais isobáricos de 850 e $700 \mathrm{hPa}$ foram estudados. Para realizar este objetivo foram empregados os dados da CFSR/NCEP.

Os valores de espessura analisados são semelhantes aqueles encontrados por (Weber, 2011) utilizando os dados de R2/NCEP. Neste estudo, diferentemente de (Weber, 2011) que considerou apenas as serras gaúcha e catarinense, separou os casos em três grupos associados à elevação em relação ao nível do mar. Os valores médios de espessura não apresentaram grande variabilidade entre os grupos. De forma geral pode-se considerar um valor limite para a ocorrência de neve a espessura representativa de $75 \%$ dos casos, ou seja, do terceiro quantil. E se comparados esses valores com os valores máximos médios de espessura para ocorrência de neve encontrados por (Heppner, 1992), apresentados na tabela 3.
Tabela 3: Comparação dos valores limite de espessura (em dam) para cada camada analisada

\begin{tabular}{ccc}
\hline Camada & \multicolumn{2}{c}{ Espessura } \\
\hline & \multicolumn{2}{c}{ P.E Heppner } \\
\cline { 2 - 3 } $500-1000$ & 551 & 543 \\
$850-1000$ & 133 & 131 \\
$700-1000$ & 289 & 285 \\
$700-850$ & 156 & 155 \\
\hline
\end{tabular}

É possível notar que os valores máximos de espessura encontrados para o hemisfério norte, são menores que os valores médios das medianas de espessura dos casos encontrados para sul do Brasil, e se assemelham a valores encontrados para apenas $25 \%$ dos casos.

A temperatura em $850 \mathrm{hPa}$, também encontrado por Heppner (1992) é em média $-9^{\circ} \mathrm{C}$ para o hemisfério norte, já no hemisfério sul a temperatura é igual a $-3^{\circ} \mathrm{C}$, porém há casos analisados com temperatura no nível de 850 $\mathrm{hPa}$ superiores a $1^{\circ} \mathrm{C}$.

A análise da água precipitável não se mostrou conclusiva do ponto de vista estatístico, uma vez que possui 
uma grande variabilidade. Além do mais o nível de 850 hPa mostra que os valores de umidade relativa ficaram abaixo de $100 \%$ e em $700 \mathrm{hPa}$ valores em torno de $40 \%$, assim, é mais fácil alcançar a saturação no nível de 850 $\mathrm{hPa}$. Logo, isso indica que a camada atmosférica relacionada a precipitação de neve, para a região sul do Brasil, seja abaixo de $850 \mathrm{hPa}$. Em função da camada de nuvens associada a precipitação de neve ser rasa, os limiares de espessura fornecidos pelas camadas entre 700-1000 hPa e 700-850 hPa não são tão significativas.

Outro ponto a se considerar para a precisão da previsão de neve no sul do Brasil é o relevo, pois a nota-se valores menores de espessura para menores latitudes, e por conseguinte é possível ocorrer precipitação de neve em latitudes mais altas com espessuras, em mais da metade dos casos, de 550 dam, ou seja, não é necessário uma camada atmosférica tão fria.

\section{Referências}

Carvalho Júnior, I. J. d. (2004). A neve em palmas/pr: Da reconstituição histórica à abordagem dinâmica. Mestrado em geografia, Universidade Estadual Paulista,São Paulo.

CIRAM (2014). Centro de informações de recursos ambientais e de hidrometeorologia de santa catarina. URLhttp://ciram.epagri.sc.gov.br/, acesso em 17 nov.2014.

Cruz, G. (2014). Eventos de neve [mensagem pessoal]. Mensagem recebida por <franciano.puhales@ufsm. br>em 28 set. 2014.

Dewey, K. F. (1977). Daily maximum and minimum temperature forecasts and the influence of snow cover. Monthly Weather Review, 105(12), 1594-1597.

Fuentes, M. V. (2009). Dinâmica e padrões da precipitação de neve no sul do Brasil. Doutorado em geofísica, Universidade Federal do Rio Grande do Sul, Porto Alegre.

G1, J. (2014). Maior nevasca de sc. Portal de Notícias G1, santa Catarina, 20 de jul. de 2014 ..

Heppner, P. O. (1992). Snow versus rain: Looking beyond the "magic" numbers. Weather and forecasting, 7(4), 683-691.

Johnston, E. C. (1995). Updated satellite technique toforecast heavy snow. Weather and forecasting, 10(2), 219-228.

McGill, R., Tukey, J. W., Larsen, W. A. (1978). Variations of box plots. The American Statistician, 32(1), 12-16.
Quadro, M. F. L. d. (2012). Estudo de vórtices ciclônicos de mesoescala associados à zona de convergência do Atlântico Sul. Doutorado em meteorologia, Universidade de São Paulo, São Paulo, disponível em:<http://www.teses.usp.br/teses/ disponiveis/14/14133/tde-06062012-115400/>.

Saha, S., Moorthi, S., Pan, H. L., Wu, X., Wang, J., Nadiga, S., Tripp, P., Kistler, R., Woollen, J., Behringer, D., et al. (2010). The ncep climate forecast system reanalysis. Bulletin of the American Meteorological Society, 91(8), 1015-1057.

Silva, V. B., Kousky, V. E., Higgins, R. W. (2011). Daily precipitation statistics for south america: An intercomparison between ncep reanalyses and observations. Journal of Hydrometeorology, 12(1), 101-117.

Weber, T. (2011). Um estudo das espessuras de diferentes camadas atmosféricas em eventos de neve no sul do Brasil. Trabalho de conclusão de curso, Bacharelado em Meteorologia, Universidade Federal de Santa Maria, Santa Maria.

Wilks, D. (2006). Statistical Methods in the Atmospheric Sciences, 2o edn. Academic Press, San Diego.

Zero, J., Hora (2007). Registro de uma nevada histórica. Zero Hora, porto Alegre, 3 de nov. de 2007. Caderno Geral, p. 30. 